\title{
Theory of systems with Constraints
}

\author{
Javier De Cruz Pérez \\ Facultat de Física, Universitat de Barcelona, Diagonal 645, 08028 Barcelona, Spain.*
}

\begin{abstract}
We discuss systems subject to constraints. We review Dirac's classical formalism of dealing with such problems and motivate the definition of objects such as singular and nonsingular Lagrangian, first and second class constraints, and the Dirac bracket. We show how systems with first class constraints can be considered to be systems with gauge freedom. We conclude by studing a classical example of systems with constraints, electromagnetic field
\end{abstract}

\section{INTRODUCTION}

In the standard case, to obtain the equations of motion, we follow the usual steps. If we use the Lagrangian formalism, first of all we find the Lagrangian of the system, calculate the Euler-Lagrange equations and we obtain the accelerations as a function of positions and velocities. All this is possible if and only if the Lagrangian of the systems is non-singular, if not so the system is non-standard and we can't apply the standard formalism. Then we say that the Lagrangian is singular and constraints on the initial data occur. Basically a constrained system is one in which there exists relations between the system's degrees of freedom that holds for all time. Constraints should not be confused with constants of motion. Constant of the motion arise as a result of the equations of motions and constraints to be restrictions on the dynamics before equation of motion are even solved. For example in the electromagnetic field (which we will see later in more detail ), the time derivative of the $A_{0}$ component of the vector potential does not appear in the action of the system, therefore the momentum conjugate to $A_{0}$ is always zero, which is a constraint . The first systematic discussion of singular Lagrian systems was given by Dirac, who developed a standard technique to "Hamiltonize" a singular Lagrangian and the new Hamiltonian formalism to obtain equations of motion for these systems.[2]

In the following sections we will describe in more detail the concepts mentioned in this introduction and describe the Dirac method. Finally we apply this formalism, to solve a classic example, the electromagnetic field. One of the most important feature of these systems is that they present gauge invariance. Therefore it can be described by a gauge theory. These are a kind of field theory in which the Lagrangian is invariant under a continious group of local transformations. We will see the relation between first class constraints and the gauge freedom. Finally we will find the Dirac brackets between the field variables (potential 4 -vector) and their conjugate momenta.

\footnotetext{
*Electronic address: jdecrupe7@alumnes.ub.edu
}

\section{GENERAL CONSIDERATIONS}

Consider a system with a finite number, $k$, of degrees of freedom is described by a Lagrangian $L=L\left(q_{s}, \dot{q}_{s}\right)$ that don't depend on $t$. The action of the system is [3]

$$
S=\int d t L\left(q_{s}, \dot{q}_{s}\right)
$$

If we apply the least action principle action $\delta S=0$, we obtain the Euler-Lagrange equations

$$
\frac{\partial L}{\partial q_{s}}-\frac{d}{d t}\left(\frac{\partial L}{\partial \dot{q}_{s}}\right)=0 \quad s=1, \ldots, k
$$

Which can be rewritten as[1]

$$
\frac{\partial^{2} L}{\partial \dot{q}_{r} \dot{q}_{s}} \ddot{q}_{s}=\frac{\partial L}{\partial q_{r}}-\frac{\partial^{2} L}{\partial q_{s} \dot{q}_{r}} \dot{q}_{s}
$$

Define the matrix $W_{r s}=\frac{\partial^{2} L}{\partial \dot{q}_{r} \partial \dot{q}_{s}}$. Expression (3) can be solved for the accelerations $\ddot{q}_{s}$ if and only if

$$
\operatorname{det}\left|W_{r s}\right| \neq 0
$$

If this determinant vanishes, then the Lagrangian is singular and the accelerations can't be solved in terms of the positions and velocities. In Hamiltonian formalism, this implies the existency of relations between $p^{\prime} s$ and $q^{\prime} s$.

\section{HAMILTONIAN TREATMENT-THE DIRAC THEORY}

The set up of a Hamiltonian formalism for a Lagrangian starts with the Legendre transformation wich defines the momenta [3]

$$
p_{s}=\frac{\partial L}{\partial \dot{q}_{s}} \quad s=1, \ldots, k
$$

Relation (5) is invertible if and only if $\operatorname{det}\left|W_{r s}\right| \neq 0$. Then they can be solved to obtain the velocities $\dot{q}_{s}$ as functions of coordinates and momenta. If the determinant vanishes the rank $W_{r s}=R<k$ and only $\mathrm{R}$ velocities can be obtained from (5) as functions of q's , p's and the remaining $(k-R)$ velocities and also that there exist 
$(k-R)$ independent relations among p's and q's. These relations, wich are direct consequences of the definitions of p's and the structure of the lagrangian.

Supose that we solve the $k-R$ constraints for the momenta [1]

$$
p_{\rho}=\xi_{\rho}\left(p_{j}, q_{s}\right) \quad s=1, \ldots, k, \quad \begin{aligned}
& j=1, \ldots, R, \\
& \rho=R+1, \ldots, k
\end{aligned}
$$

This relations are called "primary constraints", the word primary meaning that the equations of motion were not used to obtain them. If we started with $2 k$-dimensional phase-space defined by $2 k$ indpendent coordinates $q^{\prime} s$ and $p^{\prime} s$. The motion is going to be confined to a surface of lower dimensionality, defined by constraint equation. Dirac introduced the concept of "weak" and "strong" equations. Let the constrained submanifold in phase space be called $\mathrm{U}$, let $f(q, p), g(q, p)$ be two functions defined in a neighborhood of U. The values of $f$ and $g$ on $\mathrm{U}$ are obtained by replacing $p_{\rho}$ by $\xi_{\rho}$. If after this replacment $\mathrm{f}$ and $\mathrm{g}$ become equal, then we say that they are "weakly equal" and write [1]

$$
f\left(q_{s}, p_{s}\right) \approx g\left(q_{s}, p_{s}\right)
$$

Both functions $\mathrm{f}$ and $\mathrm{g}$ have a $2 \mathrm{k}$-dimensional 'gradient vector' at each point in phase space, with components $\left(\frac{\partial f}{\partial q_{s}}, \frac{\partial f}{\partial p_{s}}\right)$ and $\left(\frac{\partial g}{\partial q_{s}}, \frac{\partial g}{\partial p_{s}}\right)$ respectively. If $f$ equals $g$ on $\mathrm{U}$ and also the gradient of $f$ agrees with that of $g$ when the arguments are restricted to $\mathrm{U}$, we say that $f$ and $g$ are "strongly equal" and write

$$
f\left(q_{s}, p_{s}\right) \equiv g\left(q_{s}, p_{s}\right)
$$

The submainfold $U$ is defined by a set of weak equation. Let us define a set of functions

$$
\phi_{\rho}\left(q_{s}, p_{s}\right)=p_{\rho}-\xi\left(q_{s}, p_{j}\right)
$$

then $\mathrm{U}$ can be defined by $\phi_{\rho} \approx 0$. At this point adding the primary constraints via Lagrange mulipliers [2]

$L=p^{j} \dot{q}_{j}-H(q, p)+u^{\rho} \phi_{\rho} \quad j=1, \ldots, R \quad \rho=R+1, \ldots, k$

The action of the system defined by

$$
S=\int d t\left(p^{j} \dot{q}_{j}-H(q, p)+u^{\rho} \phi_{\rho}\right)
$$

We apply the principle of least action $\delta S=0$ and obtain [1]

$$
\begin{array}{r}
\dot{q}_{i}=\frac{\partial H}{\partial p^{i}}+u^{\rho} \frac{\partial \phi_{\rho}}{\partial p^{i}} \\
\dot{p}^{i}=-\frac{\partial H}{\partial q_{i}}+u^{\rho} \frac{\partial \phi_{\rho}}{\partial q_{i}} \\
u^{\rho}=\frac{\partial H}{\partial p_{\rho}}=\dot{q}^{\rho} \\
\phi_{\rho}=0
\end{array}
$$

We can identify arbitrary functions with velocities that we can't obtain of (5). To write the equation of motion more compactly. We introduce a mathematical operator called Poisson bracket. For two arbitrary functions $f(q, p)$ and $g(q, p)$ the Poisson bracket is defined by

$$
\{f, g\}=\frac{\partial f}{\partial q_{i}} \frac{\partial g}{\partial p^{i}}-\frac{\partial f}{\partial p^{i}} \frac{\partial g}{\partial q_{i}}
$$

With this definition the equations of motion can be written as

$$
\begin{array}{r}
\dot{q}_{i} \approx\left\{q_{i}, H\right\}+u^{\rho}\left\{q_{i}, \phi_{\rho}\right\} \\
\dot{p}_{i} \approx\left\{p_{i}, H\right\}+u^{\rho}\left\{p_{i}, \phi_{\rho}\right\}
\end{array}
$$

and, for an arbitrary function of the phase space,

$$
\dot{f} \approx\{f, H\}+u^{\rho}\left\{f, \phi_{\rho}\right\}
$$

Now let us examine the consequences of these equation of motion. We have the quantities $\dot{\phi}_{\rho}$ wich have to be zero throughout all time. We can apply the equation of motion, taking $f$ to one of the $\phi^{\prime} s$.

$$
\left\{\phi_{\sigma}, H\right\}+u^{\rho}\left\{\phi_{\sigma}, \phi_{\rho}\right\} \approx 0
$$

We must examine these conditions to see what they lead to. It is possible for then to lead directly to an inconsistency. If that happens, it would mean that original Lagrangian is such that the equation of motion are inconsistent. If the equation(11) does not have an inconsistencies, can be divided in three kinds.

One kind of equation reduces to $0=0$ and it is identically satisfied with the help of the primary constraints. We have an another kind of equation if and only if $\operatorname{det}\left\{\phi_{\sigma}, \phi_{\rho}\right\} \neq 0$ then the matrix is invertible and we can find the arbitrary functions

$$
u^{\rho} \approx-C^{\rho \sigma}\left\{\phi_{\sigma}, H\right\}
$$

Where $C^{\rho \sigma}$ is the inverse matrix of $\left\{\phi_{\sigma}, \phi_{\rho}\right\}$. In this case we can define the following expression [1]

$$
\{g, H\}^{*}=\{g, H\}-\left\{g, \phi_{\rho}\right\} C^{\rho \sigma}\left\{\phi_{\sigma}, H\right\}
$$

This expression is called Dirac bracket and is a generalization of the Poisson bracket, allowing us to write the equations of motion as

$$
\frac{d}{d t} g(q, p) \approx\{g, H\}^{*}
$$

No arbitrary function appears in the solution of the equation of motion. Let us now return to the analysis of equation (11) In general the matrix $\left\{\phi_{\sigma}, \phi_{\rho}\right\}$ is singular and the arbitrary functions $u^{\rho}$ are not all determinate. If the rank of the matrix is $M<(k-R)$ there are $(k-R-M)$ null eigenvectors $\lambda_{\sigma}^{a}$

$$
\lambda^{a \sigma}\left\{\phi_{\sigma}, \phi_{\rho}\right\} \approx 0 \quad a=1,2, \ldots,(k-R-M)
$$


Combining equations (11) and (15) we find the following further conditions on $q^{\prime} s$ and $p^{\prime} s$

$$
\lambda^{a \sigma}\left\{\phi_{\sigma}, H\right\} \approx 0 \quad a=1,2, \ldots,(k-R-M)
$$

These equations may or may not produce restrictions on the arbitrary functions. If they do not, we have produced more constraints $\zeta \approx 0$ that restrict the motion in phase space. The new constraints and all others that may yet appear, are called secondary constraints. They arise only after the equations of motion are used at least once. We apply the same procedure for secondary constraints that we apply for the primary constraints. The process ends after a finite number of steps when we have $(k-R)$ primary constraints and $A$ secondary constraints defining a submanifold $U^{\prime}$ in phase space

$$
\begin{array}{r}
\phi_{\rho} \approx 0 \quad \rho=R+1, \ldots, k \\
\zeta_{a} \approx 0 \quad a=1, . ., A
\end{array}
$$

These $A$ secondary constraints arise from the requirement that the primary ones are preserved in time. We must now add the requirement that these secondary constraints, are preserved in time as well

$$
\begin{array}{r}
\left\{\phi_{\sigma}, H\right\}+u^{\rho}\left\{\phi_{\sigma}, \phi_{\rho}\right\} \approx 0 \\
\left\{\zeta_{a}, H\right\}+u^{\rho}\left\{\zeta_{a}, \phi_{\rho}\right\} \approx 0
\end{array}
$$

And we have the matrix

$$
D=\left|\begin{array}{c}
\left\{\phi_{\rho}, \phi_{\sigma}\right\} \\
\left\{\zeta_{a}, \phi_{\sigma}\right\}
\end{array}\right|
$$

And the condition about eigenvectors

$$
\lambda^{\rho}\left\{\sigma_{\rho}, H\right\}+\lambda^{a}\left\{\zeta_{a}, H\right\} \approx 0
$$

Now we examine the D-matrix. If $\operatorname{det}|D| \neq 0$, then again we have the situation of the second kind. We can find the arbitrary functions using equation (12), and have the finally equation of motion. But if $\operatorname{det}|D|=0$ we can't do. To resolve this problem first of all we should classify the constraints. A constraint is called first class if its Poisson brackets with all constraints vanish weakly. The rest of all we call second class constraints. Note that the clasification into primary and secondary constraints refers to the origin of the constraint. And the classification into first and second class constraints refers to this properties. We call $\phi_{a}$ first class constraints ( primary or secondary ) and $\phi_{b}$ second class constraints(primary or secondary). We can express the constraints as a linear combination of these two types [2]

$$
u^{m} \phi_{m}=\eta^{a} \phi_{a}+\kappa^{b} \phi_{b}
$$

To write this expression we need a theorem that says, for an arbibtrary function $F$ that $F \approx 0$ we can express it as a linear combination of the constraints. Putting this linear combination on the equation and calculate the Dirac bracket for a $\phi_{a}$ and $\phi_{b}$, obtain

$$
\begin{aligned}
\left\{\phi_{a}, H\right\}+\eta^{a \prime}\left\{\phi_{a}, \phi_{a^{\prime}}\right\}+\kappa^{b \prime}\left\{\phi_{a}, \phi_{b^{\prime}}\right\} & \approx 0 \\
\left\{\phi_{b}, H\right\}+\eta^{a \prime}\left\{\phi_{b}, \phi_{a^{\prime}}\right\}+\kappa^{b \prime}\left\{\phi_{b}, \phi_{b^{\prime}}\right\} & \approx 0
\end{aligned}
$$

For the properties of the first class constraints the terms which survive are

$$
\begin{aligned}
\left\{\phi_{a}, H\right\} & \approx 0 \\
\left\{\phi_{b}, H\right\}+\kappa^{b \prime}\left\{\phi_{b}, \phi_{b^{\prime}}\right\} & \approx 0
\end{aligned}
$$

Now we can say that the matrix $\left\{\phi_{b}, \phi_{b}^{\prime}\right\}$ is nonsingular and therefore its inverse exists. Finally we write the equation of motion as

$$
\frac{d}{d t} g(q, p) \approx\{g, H\}+\eta^{a}\left\{g, \phi_{a}\right\}-\left\{g, \phi_{b}\right\} \gamma^{b b^{\prime}}\left\{\phi_{b}^{\prime}, H\right\}
$$

An important feature of the first class constraints is that generate a gauge transformation. Imagine that our system has only first class constraints, in this case we can't eliminate the arbitrary functions $u^{\rho}$ from the equation of motion. To resolve this situation we need fixing the gauge. This is procedure to remove the non-pysical degrees of freedom of the system. We will see an example of this in the next section

\section{EXAMPLE OF THE ELECTROMAGNETIC FIELD}

In this section we finally apply the developed theory to a physicall system, electromagnetic field. The electric and the magnetic field obey Maxwell equations. These fields are expressed in terms of the 4 -vector potential $A^{\mu}=(\phi, \vec{A})$ (we use natural units $h=c=1$ ).

$$
\begin{array}{r}
E^{i}=\partial^{i} A_{0}-\partial^{0} A^{i} \\
B_{k}=\epsilon_{k l m} \partial_{l} A_{m}
\end{array}
$$

Sumation convention understood. We can describe this field as an antisymmetric tensor with two indices [4]

$$
F_{\mu \nu}=\partial_{\mu} A_{\nu}-\partial_{\nu} A_{\mu}
$$

The Lagrangian describing this systems is

$$
L=-\frac{1}{4} F^{\mu \nu} F_{\mu \nu}
$$

An it is invariant under the following local transformation

$$
A_{\mu}^{\prime}(x)=A_{\mu}(x)+\partial_{\mu} \epsilon(x)
$$

Where $\epsilon(x)$ is an aribitrary function $(x=(t, \vec{x}))$. This means that the system has gauge invariance, and hence it may be described by a gauge theory. At this point we want to pass to the Hamiltonian formalism, for this purpose we need the Legendre Transformation, but first compute the momentum, with the following expresion [5]

$$
\pi^{\nu}=\frac{\partial L}{\partial\left(\partial_{0} A_{\nu}\right)}
$$

The momentum for $A_{0}\left(\pi^{0} \approx 0\right)$ and this is the primary constraint of the theory and the proof that the electromagnetic field is a constrained system. Additionally, we identify the canonical momentum with the electric field

$$
\pi^{i}=-F^{0 i}=E^{i}
$$


We apply the Legendre transformation $H=\pi^{\mu} \partial_{0} A_{\mu}-L$ and the Hamiltonian is

$$
H=\int d^{3} x\left(\frac{1}{4} F^{i j} F_{i j}-\frac{1}{2} \pi^{i} \pi_{i}-\partial_{i} \pi^{i} A_{0}\right)
$$

It has a functional form, for this reason we need introduce the Poisson brackets for two arbitrary functionals [5]

$$
\{F(x), G(y)\}=\int d^{3} z\left(\frac{\delta F(x)}{\delta A(z)_{\lambda}} \frac{\delta G(y)}{\delta \pi(z)^{\lambda}}-\frac{\delta F(x)}{\delta \pi(z)^{\lambda}} \frac{\delta G(y)}{\delta A(z)_{\lambda}}\right)
$$

It is, where we have introduce the functional derivative as

$$
\frac{\delta}{\delta \psi_{\mu}}=\frac{\partial}{\partial \psi_{\mu}}-\partial_{i} \frac{\partial}{\partial\left(\partial_{i} \psi_{\mu}\right)}
$$

Now, as we have described in previous sections we impose the stability of the primary constraint and apply the expression (11):

$$
\begin{array}{r}
\left\{\pi^{0}(x), H(y)\right\}+u^{1}\left\{\pi^{0}(x), \pi^{0}(y)\right\}=\left\{\pi^{0}(x), H(y)\right\} \\
=\int d^{3} z\left(\frac{\delta \pi^{0}(x)}{\delta A(z)_{\lambda}} \frac{\delta H(y)}{\delta \pi(z)^{\lambda}}-\frac{\delta \pi^{0}(x)}{\delta \pi(z)^{\lambda}} \frac{\delta H(y)}{\delta A(z)_{\lambda}}\right) \\
=-\int d^{3} z \frac{\delta H(y)}{\delta A_{\lambda}(z)} \delta_{\lambda}^{0} \delta^{3}(x-z) \\
=-\frac{\delta H(y)}{\delta A_{0}(x)}=\partial_{i} \pi^{i} \approx 0
\end{array}
$$

Therefore we obtain a secondary constraint $\partial_{i} \pi^{i} \approx 0$. This constraint is the Gauss' law in terms of the momentum field. Now we impose agains its stability. We will use the properties of the delta function to express $\partial_{i} \pi^{i}$ as a functional

$$
\partial_{i} \pi^{i}(x)=-\int d^{3} x^{\prime} \pi^{i}\left(x^{\prime}\right) \frac{\partial}{\left.\partial x^{(} i^{\prime}\right)} \delta^{3}\left(x-x^{\prime}\right)
$$

and use this expression to calculate

$$
\begin{aligned}
\left\{\partial_{i} \pi^{i}(x), H\right\} & \approx 0 \\
\left\{\partial_{i} \pi^{i}(x), \pi^{0}(y)\right\} & \approx 0
\end{aligned}
$$

The system has two first class constraint. Previously we have discussed the relationship between the first class constraints and the gauge freedom. To continue we need to fix the gauge, for instance choosing the Coulomb gauge

$$
\partial^{i} A_{i} \approx 0
$$

An repeat the process previously used

$$
\left\{\partial^{i} A_{i}, H\right\}=\partial^{i} \partial_{i} A_{0} \approx 0
$$

If we impose that the fields decay fast enough when $r \rightarrow$ $\infty\left(A_{0} \approx 0\right)$ Now we have four constraints .

$$
\begin{array}{cl}
\phi_{1}=\pi^{0} \approx 0 & \phi_{2}=\partial_{i} \pi^{i} \approx 0 \\
\phi_{3}=A_{0} \approx 0 & \phi_{4}=\partial^{i} A_{i} \approx 0
\end{array}
$$

In principle we had one primary constraint of the first class and one secondary constraint of the first class. The gauge fixing convert the first class into a second class. And now we have one primary constraint of second class and three secondary constraints of second class. Now let's make a change of coordinates, using a Fourier transform, and work in the momentum space [4]

$$
\begin{aligned}
& A_{i}(\vec{x}, t)=\frac{1}{(2 \pi)^{\frac{3}{2}}} \int d^{3} k \tilde{A}(\vec{k}, t) i e^{i \vec{k} \vec{x}} \\
& \pi_{i}(\vec{x}, t)=\frac{1}{(2 \pi)^{\frac{3}{2}}} \int d^{3} k \tilde{\pi}_{i}(\vec{k}, t) e^{i \vec{k} \vec{x}}
\end{aligned}
$$

It is straightforward to prove that with this change of coordinates the constraints take the following form

$$
\begin{aligned}
\phi_{1}(\vec{k})=\tilde{\pi^{0}}(\vec{k}) \approx 0 & \phi_{2}(\vec{k})=k_{i} \tilde{\pi}^{i}(\vec{k}) \approx 0 \\
\phi_{3}(\vec{k})=\tilde{A}_{0}(\vec{k}) \approx 0 & \phi_{4}(\vec{k})=k^{i} \tilde{A}_{i}(\vec{k}) \approx 0
\end{aligned}
$$

We can make the following decoposition

$$
\begin{gathered}
\tilde{\vec{A}}=\tilde{A}_{\| \mid} \frac{\vec{k}}{|\vec{k}|}+\tilde{\vec{A}}_{\perp} \\
\tilde{\vec{\pi}}=\tilde{\pi}_{\|} \frac{\vec{k}}{|\vec{k}|}+\tilde{\vec{\pi}}_{\perp}
\end{gathered}
$$

Where $\tilde{A}_{\|}=\left(k^{i} \tilde{A}_{i}\right)$ and $\tilde{\pi}_{\|}=\left(k_{i} \tilde{\pi}^{i}\right)$. Thus rewrite the second and fourth constraints as $\phi_{2}(\vec{k})=\tilde{\pi}_{\|}(\vec{k}) \approx 0$ and $\phi_{4}(\vec{k})=\tilde{A}_{\|}(\vec{k}) \approx 0$. Therefore, $\tilde{\vec{A}}_{\perp}$ can be written as a linear combination of two unit vectors perpendicular to $\vec{k}$

$$
A_{i}(\vec{x}, t)=\frac{1}{(2 \pi)^{\frac{3}{2}}} \int d^{3} k e^{i \vec{k} \vec{x}}\left(\sum_{a=1,2} A_{a}(\vec{k}, t) \hat{e}_{a i}\right)
$$

Same procedure for the momentum. In position space the Hamiltonian has the following expression in terms of $\vec{E}$ and $\vec{B}$

$$
H=\frac{1}{2} \int d^{3} x\left(\vec{E}^{2}+\vec{B}^{2}\right)
$$

We want express the Hamiltonian in the momentum space, for this purpose, we use the Parseval's identity [4]

$$
\frac{1}{2} \int d^{3} x\left(\vec{E}^{2}+\vec{B}^{2}\right)=\frac{1}{2} \int d^{3} k\left(\tilde{\vec{E}} \tilde{\vec{E}}^{*}+\tilde{\overrightarrow{\vec{B}}} \tilde{\vec{B}}^{*}\right)
$$

Using the relations $\tilde{E}^{i}=\tilde{\pi}^{i}, \tilde{B}_{k}=i \epsilon_{k l m} k_{l} \tilde{A}_{m}=$ we can write the Hamiltonian as

$$
H=\frac{1}{2} \int d^{3} k\left(|\tilde{\vec{\pi}}|^{2}+|\vec{k}|^{2}|\tilde{\vec{A}}|^{2}\right)
$$

Now the Hamiltonian takes the form of the superposition of an infinity of decoupled oscillators of frequency $\omega=$ 
$|\vec{k}|$. Note that we have not first class constraints, this fact allows us to write the matrix $M\left(k, k^{\prime}\right)=\left|\left\{\phi_{\rho}, \phi_{\sigma}\right\}\right|$ of the constraints and compute his inverse. First compute the elements of $M\left(k, k^{\prime}\right)$

$$
\begin{array}{r}
\left\{\tilde{\pi}^{0}(k), \tilde{A}_{0}\left(k^{\prime}\right)\right\}=-\delta^{3}\left(k+k^{\prime}\right) \\
\left\{\tilde{\pi}^{0}(k), \tilde{A}_{\|}\left(k^{\prime}\right)\right\}=0 \\
\left\{\tilde{\pi}_{\|}(k), \tilde{A}_{\|}\left(k^{\prime}\right)\right\}=-k^{2} \delta^{3}\left(k+k^{\prime}\right) \\
\left\{\tilde{\pi}_{\|}(k), \tilde{A}_{0}\left(k^{\prime}\right)\right\}=0
\end{array}
$$

And obtain the expression for the M-matrix

$$
M\left(k, k^{\prime}\right)=\left(\begin{array}{cccc}
0 & 0 & -1 & 0 \\
0 & 0 & 0 & -k^{2} \\
1 & 0 & 0 & 0 \\
0 & k^{2} & 0 & 0
\end{array}\right) \delta^{3}\left(k+k^{\prime}\right)
$$

The problem of findig the inverse of the M-matrix reduces to calculate the components of the $M^{-1}\left(k, k^{\prime}\right)$ that match the relationship

$$
\int d^{3} k^{\prime \prime} M\left(k, k^{\prime \prime}\right)_{\rho \alpha} M^{-1}\left(k^{\prime \prime}, k^{\prime}\right)^{\alpha \sigma}=\delta_{\rho}^{\sigma} \delta^{3}\left(k+k^{\prime}\right)
$$

The only nonvanishing matrix elements are $M_{31}^{-1}=$ $-\delta^{3}\left(k+k^{\prime}\right)$ and $k^{2} M_{42}^{-1}=-\delta^{3}\left(k+k^{\prime}\right)$ recall that $M_{\rho \sigma}$ is an antisymetric matrix. So the inverse of the M-matrix becomes

$$
M^{-1}\left(k, k^{\prime}\right)=\left(\begin{array}{cccc}
0 & 0 & 1 & 0 \\
0 & 0 & 0 & k^{-2} \\
-1 & 0 & 0 & 0 \\
0 & -k^{-2} & 0 & 0
\end{array}\right) \delta^{3}\left(k+k^{\prime}\right)
$$

The existence of the inverse matrix proves that the gauge conditions really fix the gauge completly. Having calculated the inverse, we are now able to write down the Dirac brakets using(13)

$$
\begin{gathered}
\left\{F(k), G\left(k^{\prime}\right)\right\}^{*}=\left\{F(k), G\left(k^{\prime}\right)\right\} \\
-\int d^{3} k^{\prime \prime} d^{3} k^{\prime \prime \prime}\left\{F(k), \phi_{\alpha}\left(k^{\prime \prime}\right)\right\}\left(M^{-1}\right)^{\alpha \beta}\left\{\phi_{\beta}\left(k^{\prime \prime \prime}\right), G\left(k^{\prime}\right)(44)\right.
\end{gathered}
$$

We use this expression to calculate the fundamental Dirac brackets $\mu, \nu=0,1,2,3 i, j=1,2,3$

$$
\begin{array}{r}
\left\{\tilde{A}_{\mu}(k), \tilde{A}_{\nu}\left(k^{\prime}\right)\right\}^{*}=\left\{\tilde{\pi}^{\mu}(k), \tilde{\pi}^{\nu}\left(k^{\prime}\right)\right\}^{*}=0 \\
\left\{\tilde{A}_{i}(k), \tilde{\pi}^{j}\left(k^{\prime}\right)\right\}^{*}=\left(\delta_{i}^{j}-\frac{k_{i} k^{j}}{k^{2}}\right) \delta^{3}\left(k+k^{\prime}\right)
\end{array}
$$

For the components of transverse components $\tilde{A}_{\alpha}(k)=$ $\tilde{A}_{i} \hat{e}_{\alpha}^{i}$ and $\tilde{\pi}_{\alpha}(k)=\tilde{\pi}^{i} \hat{e}_{i \alpha}$ we have

$$
\begin{array}{r}
\left\{\tilde{A}_{\alpha}(k), \tilde{A}_{\beta}\left(k^{\prime}\right)\right\}^{*}=\left\{\tilde{\pi}^{\alpha}(k), \tilde{\pi}^{\beta}\left(k^{\prime}\right)\right\}^{*}=0 \\
\left\{\tilde{A}_{\alpha}(k), \tilde{\pi}^{\beta}\left(k^{\prime}\right)\right\}^{*}=\delta_{\alpha}^{\beta} \delta^{3}\left(k+k^{\prime}\right)
\end{array}
$$

And calculate the equation of motion for components that not subjects to restrictions

$$
\begin{array}{r}
\left\{\tilde{A}_{\alpha}(k), H\right\}^{*}=\tilde{\pi}_{\alpha}(k) \\
\left\{\tilde{\pi}_{\alpha}(k), H\right\}^{*}=-|\vec{k}|^{2} \tilde{A}_{\alpha}(k)
\end{array}
$$

\section{CONCLUSIONS}

In this paper we study a systems with constraints and develop a consistent method for the dynamical evolution of such systems. To obtain the Hamiltonian of the system starting from the Lagrangian, required a Legendre transformation, where the canonical momentum had to be introduced. If the Lagrangian is singular, when do the process, arise a certain relations between the phase space variables. These relations impose a conditions abaout dynamical of the system and are therefore constraint functions. We have also classified the constraints according to their origin or their properties and we see the relation between first class constraint ant the gauge freedom. To write the equation of motion we use the Dirac bracket. This operator is built with the Poisson brackets and constraints matrix. Finally we apply all this theory to resolve the problem of find the equation of motion of the electromagnetic field. To eliminate the non-physical degrees of freedom we have fix the gauge, this has generated more restriccions abaout the system. When we apply all the constraints, only two componnts of the four potential and other two of momentum remain independent. The final equations of motion are consistent with all constraints.

\section{Acknowledgments}

I would like to express my gratitue to my advisor Josep LLosa for helping me in evertyhing I needed, and dedicate many hours of his time.
[1] E.C.G ShudarshanClassical dynamics : A modern perspective (John-Wiley\& Sons, New York 1974).

[2] P.A.M.Dirac Lectures on quantum mechanics Belfer Graduate School of Science,1964.

[3] H.Goldstein Mecánica Clásica Editorial Reverté ,1987
[4] J.Llosa,A.Molina Relativitat especial amb aplicacions a l'electrodiàmica clàssica, 2a edició

[5] Weinberg The Quantum theory of fields volume I, 1995 